\title{
Effect of NHS breast screening programme on mortality from breast cancer in England and Wales, 1990-8: comparison of observed with predicted mortality
}

\author{
R G Blanks, S M Moss, C E McGahan, M J Quinn, P J Babb
}

\begin{abstract}
Objective To assess the impact of the NHS breast screening programme on mortality from breast cancer in women aged 55-69 years over the period 1990-8.

Design Age cohort model with data for 1971-89 used to predict mortality for 1990-8 with assumption of no major effect from screening or improvements in treatment until after 1989. Effect of screening and other factors on mortality estimated by comparing three year moving averages of observed mortality with those predicted (by five year age groups from 50-54 to 75-79), the effect of screening being restricted to certain age groups.

Setting England and Wales.

Subjects Women aged 40 to 79 years.

Results Compared with predicted mortality in the absence of screening or other effects the total reduction in mortality from breast cancer in 1998 in women aged 55-69 was estimated as 21.3\%. Direct effect of screening was estimated as $6.4 \%$ (range of estimates from 5.4-11.8\%). Effect of all other factors (improved treatment with tamoxifen and chemotherapy, and earlier presentation outside the screening programme) was estimated as $14.9 \%$ (range 12.2-14.9\%).

Conclusions By 1998 both screening and other factors, including improvements in treatment, had resulted in substantial reductions in mortality from breast cancer. Many deaths in the 1990s will be of women diagnosed in the 1980s and early 1990s, before invitation to screening. Further major effects from screening and treatment are expected, which together with cohort effects should result in further substantial reductions in mortality from breast cancer, particularly for women aged 55-69, over the next 10 years.
\end{abstract}

\section{Introduction}

The NHS breast screening programme was introduced in England and Wales in 1988, on the recommendations of the Forrest committee. ${ }^{1}$ These recommendations were based on the findings of randomised controlled trials, which showed that mammography could reduce mortality from breast cancer in women aged 50 years and over by $25-30 \%$ over a period of about 10 years. In England and Wales women aged 50-64 are invited for screening every three years. Evidence from the programme itself indicates, and national incidence rates confirm, that the build up of activity was gradual and that the "prevalent" round of screening was not completed until 1995.

In 1992 the Department of Health set a target for breast cancer of a reduction in mortality of $25 \%$ in the age group invited for screening by the year $2000{ }^{2}$ It was subsequently acknowledged that this target should be applied to the age group 55-69 years. Screening would not be expected to affect mortality in the 50-54 years age group because the average age at first screening for women in the programme is 51.5 years (as women are first invited between the ages of 50 and 52), and in the randomised controlled trials there was little or no effect of screening in the first four years. Also survival from breast cancer in the late 1980s was good (five year relative survival rate was almost $70 \%$ and 10 year survival over $50 \%)^{3}$ and will have been higher in women detected by screening because of earlier detection during the preclinical phase. The programme has set targets relating to uptake and rates of cancer detection, which, if achieved, should eventually lead to the target reduction in mortality of $25 \%{ }^{4}$

The targets for cancer detection are based on the detection rates observed in the Swedish two county randomised controlled trial. ${ }^{5}$ In the analysis of this trial, all deaths from breast cancer in women with date of diagnosis before the date of entry to the trial in both the study and control arms were excluded. In 1989, Day suggested that the reduction in mortality from breast cancer in the target population should be at least 25\% after 10 years from the start of screening, but only in those women free from breast cancer when first invited to screening. ${ }^{6}$ National mortality statistics will include both women who were and were not free from breast cancer at the time of their first invitation. The further away in time from the start of screening, the greater will be the proportion of women in the former category. Many deaths from breast cancer in the 1990s will be in women diagnosed with breast cancer before any invitation to screening, as full coverage of the population of England and Wales did not occur until 1995. Consequently the impact of screening on breast cancer on the national mortality statistics by the year 2000 is likely to be much less than $25 \%{ }^{2}$

Mortality from breast cancer began falling in England and Wales from around 1990, before the programme could have been expected to have a major impact. ${ }^{7}$ By 1994, in the age group 55-69 years, where mortality would be affected by screening, there was a $12 \%$ reduction compared with prescreening rates in the late 1980s. An explanation for some of this reduction may be the use of adjuvant tamoxifen, which by 1990 was in widespread use for women aged over 50 years. It has been pointed out, however, that there is no direct evidence for such an effect and that by 1993 some reduction in mortality (albeit rather small) would have been expected from screening. ${ }^{8}$

Breast cancer mortality in this period may also have been affected by changes in stage at presentation, possibly because of increased publicity about breast cancer during the introduction of the screening programme, and by birth cohort effects. ${ }^{9}$ Cohort effects

\section{Editorial by \\ Nystrøm \\ Cancer Screening \\ Evaluation Unit, \\ Institute of Cancer \\ Research, Section of \\ Epidemiology, \\ Sutton, Surrey \\ SM2 $5 \mathrm{NG}$ \\ R G Blanks \\ epidemiologist \\ S M Moss \\ associate director \\ C E McGahan \\ statistician \\ Office for National \\ Statistics, \\ Demography and \\ Health Division, \\ London \\ SW1V 2QQ \\ M J Quinn \\ director, National \\ Cancer Intelligence \\ Centre \\ P J Babb \\ epidemiologist \\ Correspondence to: \\ S M Moss \\ s.moss@icr.ac.uk}

BMJ 2000;321:665-9 
may cause mortality to increase or decrease in different age groups and result in the true reduction attributable to screening and improvements in treatment being over or underestimated. The term "improvements in treatment" is used here to include effects of both changes in treatment and other factors including earlier presentation outside the age range of women invited for screening and structural changes in the NHS after the Calman-Hine report. ${ }^{10}$

We used an age cohort model based on mortality data for 1971-89 (which would not have been affected by screening) to predict the mortality from breast cancer for 1990-8. By comparing the observed mortality in different age groups with that predicted by the model we estimated the separate effects of screening and of improvements in treatment and other factors.

\section{Method}

We obtained data on mortality from breast cancer for England and Wales from the database of deaths at the Office for National Statistics for 1971-99 by single year of age and calendar year of death. We reconstructed these data into five year age groups (from 40-44 to 75-79 years) and cohort groups from 1896 to 1946 . We corrected the annual numbers of deaths for coding changes that occurred in 1984 and 1993 as a result of revised interpretation of $\mathrm{WHO}$ rule 3 and for other procedural changes in 1993; the overall adjustment was only a few percentage points for women aged 50-74 but greater for younger and older age groups. ${ }^{7}$

We assumed that use of tamoxifen, other treatment changes, and screening would not have had any substantial impact on mortality until 1990 and that age and birth cohort effects were the major influences on mortality from 1971 to 1989 .

We fitted an age cohort model using five year age groups from 40-44 to 75-79 for 1971-89 using log linear Poisson modelling with the generalised linear models command in the statistical package STATA. Although we considered that an age cohort model was likely to give the best fit we also used age (alone) and age period models for comparison, according to the methods of Clayton and Shifflers. ${ }^{11}$ The age cohort model was highly significant $(\mathrm{P} \ll 0.001)$ when tested against the age model alone. Other models, such as an age period model did not fit the data well. Although the age cohort model provided a good fit to the data, the 1946 cohort estimate was based on only few data and had wide confidence limits. To provide a better estimate for this cohort we extrapolated the rate ratios for the 1896 to 1941 cohorts to 1946 by fitting a quadratic model, after noting that the rate ratios increased up to the 1926 cohort and decreased thereafter. The final model was used to predict the mortality from breast cancer in the absence of screening or treatment effects for 1990-8 for each five year age group.

From the mid-1980s the use of tamoxifen in women with breast cancer increased rapidly. By 1990 in the Thames regions over $90 \%$ of women aged over 50 years and over $50 \%$ below the age of 50 received tamoxifen. ${ }^{12}$ Results of randomised controlled trials suggest a fairly uniform effect of tamoxifen across the age range 50-79 years. ${ }^{13}$ The effect of screening, in contrast, will be more age dependent because of the age range of women invited. Screening will reduce mortality in older age groups as time progresses, influencing mortality in women aged 55-74 by 2000 and those aged 55-79 by 2005 .

Three year moving averages of mortality were used to increase statistical stability: the results in table 1 for 1998 are based on the average mortality for 1997 , 1998, and 1999. We compared the observed mortality from national statistics from 1990 to 1998 with that predicted by the model for each five year age group from 50-54 to 75-79. For 1990-5 we assumed mortality in age groups 50-54, 70-74, and 75-79 to be affected only by factors other than screening, and assumed mortality in age groups 55-59, 60-64 and 65-69 to be affected by these factors plus screening. After 1995 mortality in the 70-74 age group will have been partly affected by screening (the only age groups not affected by screening are 50-54 and 75-79).

Once the baseline predictions of what the mortality would have been over the period 1990-8 in the absence of screening are established, our method of apportioning any reduction in mortality between screening and other factors is deterministic. It is therefore not possible to put conventional confidence intervals around the two proportions. We therefore explored the sensitivity of our results to changes in the age cohort model by considering two alternative sets of predicted mortality. Firstly, we assumed that mortality in each age group would have remained at its rate before screening-that is, the rates in each year from 1990 to 1998 inclusive were taken to be the same as those in 1989. This is more neutral than the cohort effects predicted by our model, which give decreasing rates in women up to the 60-64 age group and increasing rates in those over 70 (see below and fig 1). Secondly, we assumed that the curvature in the cohort rate ratios (see table in appendix) was more marked (by $5 \%$ points in the youngest and oldest cohorts, pro rata for the others) giving a more extreme divergence in predicted mortality by age group.

\section{Results}

Figure 1 shows the observed mortality from breast cancer from 1971 to 1999 by five year age group (solid line) together with the modelled rates from 1990 to 1998 (dotted lines). Throughout the whole period 1971 to 1998 , mortality was higher in each successive age group. Mortality in all age groups increased after 1971 , but by the early 1980 s the rate in the youngest women (50-54 years) had more or less stabilised. Mortality began to fall soon after screening started; the falls seem to have been larger in women aged 55-69 years than in the other age groups. Details of the model estimates are shown in the table in the appendix. The cohort rate ratios show that mortality from breast cancer peaked in women in the 1926 cohort, with a declining risk in later cohorts. The model predicts declining mortality in women under 65 years for 1990-8 but an increasing rate in women over 70 .

Figure 2 shows the data by year of birth rather than year of death for age groups 50-54 (in which mortality will be affected only by improvements in treatment), 55-69 (affected by screening and improvements in treatment), and 75-79 (affected only by improvements in treatment). Deaths in 1971 to 1989 have been distinguished from those in 1990 to 1998 . The age 


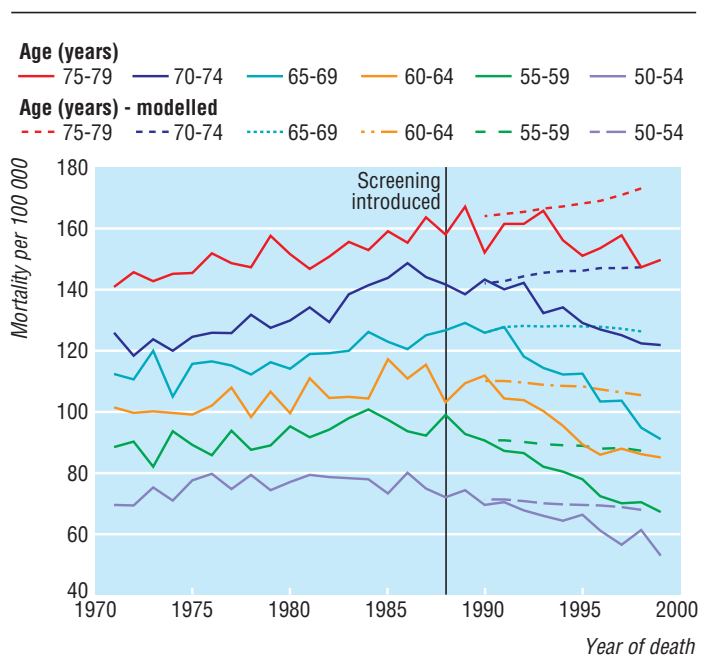

Fig 1 Mortality from breast cancer by year of death for selected age groups, England and Wales, 1971-99

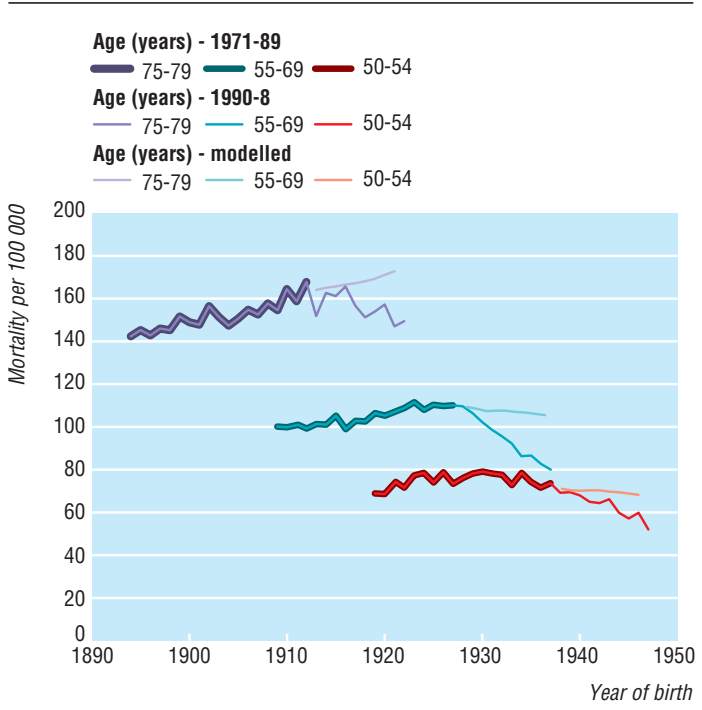

Fig 2 Mortality from breast cancer by year of birth for selected age groups, England and Wales, 1971-99

group 70-74 has been omitted because women will have been affected by screening from 1995. The trends in mortality, albeit at different levels, in the three age groups were similar until screening was introduced. Thereafter, mortality in women aged 55-69 dropped considerably more than in the other two age groups.

Table 1 compares observed mortality with that predicted by the model for the two years 1993 and 1998 . By 1993 only a small direct effect of screening would be expected; 1998 is the last complete year for which three year moving average data are available. The average reduction in mortality in 1993 in age groups $50-54,70-74$, and $75-79$ was $5.6 \%$. This is assumed to be the contribution from improvements in treatment. In the age groups 55-59, 60-64, and 65-69 the average reduction was $8.8 \%$, attributable to both treatment and screening. The estimated reduction in mortality directly from screening is therefore $3.2 \%$. In 1998 the average reduction in mortality in age groups 50-54 and $75-79$ had increased to $14.9 \%$ and that in age groups $55-59,60-64$, and $65-69$ to $21.3 \%$, giving an estimated direct contribution from screening of $6.4 \%$.

The overall reductions in mortality in 1998 from the two alternative predictions of mortality are shown in table 2. The corresponding direct effects of screening were $11.8 \%$ and $5.4 \%$ and of improvements in treatment were $12.2 \%$ and $12.6 \%$. Consequently, the best estimate of the direct effect of screening is a $6.4 \%$ reduction in mortality with a range of estimates of 5.4$11.8 \%$, and the best estimate for improvements in treatment is $14.9 \%(12.2-14.9 \%)$.

\section{Discussion}

From 1990 to 1998 our analyses show a reduction of up to $21 \%$ in mortality from breast cancer that is directly attributable to improvements in treatment, earlier presentation outside of screening, and screening itself, after allowance for cohort effects and changes of coding for breast cancer.

By 1998 the NHS breast screening programme had directly produced a reduction in annual mortality from breast cancer of 6-7\% in women aged 55-69, although the sensitivity analysis indicated a probable range of $5-12 \%$. The best estimate of number of deaths from breast cancer in the 55-69 year age group prevented by screening in 1998 was 320 (range 270 to 590). The number of deaths prevented by screening is expected to increase over the coming years as from 1995 all women aged 50-64 were invited for screening. The expected further effects from screening, together with improved treatments such as the use of tamoxifen for five years and the impact of cohort effects all suggest that mortality in women aged 55-69 will continue to decrease over the next 10 years.

\section{Methodological difficulties}

There are several inherent problems that limit the precision of our estimate of the reduction in mortality resulting from screening. These include errors inherent in projecting mortality into future years, such

Table 1 Modelled and observed mortality (rates based on three year averages) from breast cancer per 100000 in 1993 and 1998 by age group

\begin{tabular}{|c|c|c|c|c|c|c|}
\hline \multirow{2}{*}{$\begin{array}{l}\text { Age group } \\
\text { (years) }\end{array}$} & \multicolumn{3}{|c|}{1993} & \multicolumn{3}{|c|}{1998} \\
\hline & Modelled & Observed & \% Difference & Modelled & Observed & \% Difference \\
\hline $50-54$ & 70.0 & 65.7 & -6.1 & 68.1 & 56.5 & -17.0 \\
\hline $55-59$ & 88.9 & 82.6 & -7.1 & 86.9 & 68.6 & -21.1 \\
\hline $60-64$ & 109.1 & 99.5 & -8.8 & 105.2 & 85.4 & -18.8 \\
\hline $65-69$ & 127.7 & 114.3 & -10.5 & 126.4 & 96.1 & -24.0 \\
\hline $70-74$ & 145.5 & 134.8 & -7.4 & $(147.5)^{\star}$ & $(122.8)^{\star}$ & $(-16.7)^{\star}$ \\
\hline $75-79$ & 166.4 & 160.9 & -3.3 & 173.3 & 151.1 & -12.8 \\
\hline
\end{tabular}

${ }^{*}$ Numbers shown in brackets because age group 70-74 not used for estimation of effect of screening.

Table 2 Modelled and observed mortality (rates based on three year averages) from breast cancer per 100000 in 1998 by age group: two alternative estimates

\begin{tabular}{|c|c|c|c|c|c|c|}
\hline \multirow[b]{2}{*}{$\begin{array}{l}\text { Age group } \\
\text { (years) }\end{array}$} & \multicolumn{3}{|c|}{1989 as baseline } & \multicolumn{3}{|c|}{ Increased curvature of cohort rate ratios } \\
\hline & $\begin{array}{c}\text { Observed } \\
1989\end{array}$ & $\begin{array}{c}\text { Observed } \\
1998\end{array}$ & $\%$ Difference & Modelled & Observed & $\%$ Difference \\
\hline $50-54$ & 71.6 & 56.5 & -21.1 & 63.8 & 56.5 & -11.4 \\
\hline $55-59$ & 94.3 & 68.6 & -27.2 & 82.2 & 68.6 & -16.5 \\
\hline $60-64$ & 108.2 & 85.4 & -21.1 & 101.3 & 85.4 & -15.7 \\
\hline $65-69$ & 125.9 & 96.1 & -23.7 & 122.9 & 96.1 & -21.8 \\
\hline $70-74$ & 136.8 & 122.8 & -10.3 & $(147.2)^{\star}$ & $(122.8)^{*}$ & $(-16.6)^{*}$ \\
\hline $75-79$ & 159.3 & 151.1 & -5.1 & 175.1 & 151.1 & -13.7 \\
\hline
\end{tabular}

${ }^{*}$ Numbers shown in brackets because age group 70-74 not used for estimation of effect of screening. 
as the fit of the model and the greater instability in the numbers of deaths in younger age groups being projected forward in time to predict rates in older women. The age cohort model has, however, also been found by others to be the best model, and the rate ratios from our model are closely similar. ${ }^{14}$ Our assumption that an age cohort model would still have fitted the data in 1990 to 1998 in the absence of improvements in treatment and screening effects is impossible to test; but the potential errors in the observed rates, which are each based on an average of three years' data, will be relatively small. It could be argued that screening affects the older women in the age range 50-54; if the only group considered to be unaffected by screening is 75-79 years, the direct reduction in mortality from screening in 1998 increases from $6.4 \%$ to $8.5 \%$. Our analyses using alternative predictions of mortality for 1990-8 have led to estimates of the mortality reduction from screening of between $5.4 \%$ and $11.8 \%$. This range indicates the limitations in our methods, but clearly shows that the mortality in women aged 55-69 has decreased more than in the adjacent age groups and that this is directly related to the screening programme.

\section{Population screening and randomised controlled trials}

It is important to emphasise that deaths in women diagnosed with breast cancer before invitation to screening will cause a severe dilution of the effect of the screening programme on national mortality statistics, particularly in the first decade of screening, compared with the results from randomised controlled trials. The extent and timing of a reduction in mortality due to screening will depend on several factors. These include the proportion of deaths from breast cancer in a given year occurring in women diagnosed with the disease before their first invitation to screening and the time period over which $100 \%$ coverage of the target population is achieved. Given the relatively good survival rates in the 1980 s and that there is excess mortality in women with breast cancer 15-20 years after diagnosis $^{1516}$ a large number of the deaths that occurred in the 1990 s would have been in women who were diagnosed with breast cancer before invitation to screening. If we take these factors into account and allow for the fact that in the first years of screening the sensitivity of the process in detecting cancers ${ }^{17}$ was some $25 \%$ lower than anticipated on the basis of the Swedish two county study, ${ }^{5}$ it is likely to be between 2005 and 2010 before the full effect of screening is seen in national breast cancer mortality statistics.

Results from a computer simulated model (MISCAN) suggested that $70 \%$ of the reported $12 \%$ reduction in mortality seen by $1994-$ that is, about $8 \%$-may be due to screening. ${ }^{18}$ Our lower estimated reductions due to screening may be consistent with the lower sensitivity of the screening programme in the early years of screening. ${ }^{17}$ The earlier "shortfall" in detection of invasive cancer in the programme was mostly due to a low sensitivity for small invasive cancers-those least likely to have metastasised and the most important to detect to achieve a high reduction in mortality. The "steady state" effect of screening, if we assume the same sensitivity and improvement in prognosis as achieved in the Swedish two county study and an uptake of around $70 \%$, has been estimated as a $25 \%$ reduction in mortality from breast cancer in women aged 55-69. ${ }^{18}$

There are several reasons why the effect of screening in the population screening programme could be different from that observed in the Swedish two county randomised controlled trial. The stage of breast cancers in women diagnosed before the introduction of screening in England and Wales may have differed from that in the control group of the Swedish two county study. A tendency towards a late stage at presentation would in theory result in a greater potential for the effect of earlier diagnosis. In addition, the effect of screening on mortality is likely to vary according to the treatment of early stage disease and in particular the extent to which tamoxifen is used.

\section{Treatment and other effects}

We estimated the effect of tamoxifen and other factors, excluding the direct effect of screening by 1998 , to have been a reduction in mortality of between $12.2 \%$ and $14.9 \%$. The randomised controlled trials for tamoxifen often report on early stage disease in women who are positive for oestrogen receptor. As about one third of women are negative for oestrogen receptor, and many women with breast cancer do not present with early stage disease, the benefit of tamoxifen to the population of women with breast cancer is difficult to predict from the results of randomised controlled trials. Peto has suggested that the absolute benefit produced by a few years of adjuvant tamoxifen for patients with breast cancer is not large $(50 \%$ survival may be increased to $55 \%$ or $60 \%),{ }^{19}$ but the treatment is widely given and the disease is common. Improvements in chemotherapy and other factors, including structural changes in the NHS, will also have contributed to this reduction in mortality. Recent work has suggested an additional effect of the massive publicity surrounding breast cancer whereby since the mid-1980s there has been a shift to earlier diagnosis of tumours. ${ }^{9}$ This may explain why mortality from breast cancer decreased so rapidly in the early 1990s with treatment and earlier presentation outside the screening programme together with cohort effects in women under age 70 all contributing to the apparent falls.

\section{Improvements in screening}

Since the early years of screening there have been substantial improvements in sensitivity, particularly for small invasive cancers, ${ }^{20}$ as a result of the increased use of two view mammography, the use of higher film densities, and increasing experience of radiologists.

\section{Conclusions}

There has been considerable recent debate over the attribution of the reduction in mortality from breast cancer to improvements in treatment or screening. The purpose of screening is to detect cancers at an earlier stage, when treatment is more effective. Our research indicates that for 1990-8 improvements in treatment and screening both probably have major roles in the reduction in mortality from breast cancer. The difficulties in producing quantitative estimates of the effect of a national screening programme (or improvements in treatment) on mortality are well recognised.$^{18}$ Such difficulties illustrate the importance of carrying out well conducted randomised controlled trials before the introduction of population based screening. 


\section{What is already known on this topic}

Screening for breast cancer with mammography has been shown to reduce mortality by $25-30 \%$ in randomised controlled trials

Women in England and Wales started to be invited for screening between 1988 and 1995

\section{What this study adds}

Analysis of the mortality from breast cancer in England and Wales between 1971 and 1999 shows that between 1990 and 1998 there was a real fall of $21.3 \%$ in women aged $55-69$

Of this fall, $6.4 \%$ has been attributed to screening and $14.9 \%$ to improvements in treatment and other factors

The effect of screening on national statistics has been slower to take effect compared with randomised controlled trials partly because many deaths from breast cancer in the 1990s will be in women diagnosed before any invitation to screening

Substantial improvements in screening and the decreasing proportion of deaths of women in whom breast cancer was diagnosed before screening will lead to a continuing decline in mortality in women aged 55-69 over the next ten years

Continuous monitoring of the performance of the NHS breast screening programme is essential. Recent results of such monitoring suggest that the effect of the programme on mortality will increase substantially in future years. ${ }^{20}$

We thank Professor N E Day and Professor M Vessey and the referees for comments on earlier drafts of this paper.

Funding: The Cancer Screening Evaluation Unit receive support from the Department of Health; the views expressed in this paper are those of the authors and not necessarily those of the Department of Health.

Competing interests: None declared.

Contributors: SM and RB initiated the research. RB and $\mathrm{CMcG}$ carried out the analysis of the data. The paper was written jointly by $\mathrm{SM}, \mathrm{RB}, \mathrm{MQ}$ and $\mathrm{PB} . \mathrm{MQ}$ and $\mathrm{PB}$ were responsible for supplying the data and advising on the analysis. SMM is guarantor for the paper.

1 Forrest APM. Breast cancer screening: report to the health ministers of England, Wales, Scotland and Northern Ireland. London: HMSO, 1986

2 Department of Health. The health of the nation: a strategy for health in England. London: HMSO, 1992

3 Coleman MP, Babb P, Damiecki P, Groscalude P, Honjo S, Jones J, et al. Cancer survival trends in England and Wales 1971-1995: deprivation and NHS region. London: Stationery Office, 1999. (Studies in medical population subjects No 61.)

4 Moss S, Blanks R, for the Interval Cancer Working Group. Calculating appropriate target cancer detection rates and expected interval cancer rates for the UK NHS breast screening programme. J Epidemiol Community Health 1998;52:111-5.

5 Tabar L, Fagerberg CJ, Gad A, Baldetorp L, Holmberg LH, Grontoft O, et al. Reduction in mortality from breast cancer after mass screening with mammography. Randomised trial from the breast cancer screening working group of the Swedish National Board of Health and Welfare working group of the
Lancet 1985;i:829-32.

6 Day NE, Williams DRR, Khaw KT. Breast cancer screening programmes: the development of a monitoring and evaluation system. Br J Cance 1989;59:954-8.

7 Quinn M, Allen E, on behalf of the United Kingdom Association of Cancer Registries. Changes in incidence of and mortality from breast cancer in England and Wales since introduction of screening. BMJ 1995;311:1391-5.

\section{Appendix}

Multiplicative age cohort model with Poisson regression used to estimate mortality from breast cancer, 1990-8

\begin{tabular}{lc}
\hline & Rate ratio $\mathbf{( 9 0 \%} \mathbf{~ C l )}$ \\
\hline Age group (years): & $0.42(0.41$ to 0.43$)$ \\
\hline $40-44$ & $0.71(0.70$ to 0.73$)$ \\
\hline $55-49$ & 1.00 (baseline) \\
\hline $50-54$ & $1.23(91.21$ to 1.25$)$ \\
\hline $60-64$ & $1.45(1.42$ to 1.47$)$ \\
\hline $65-69$ & $1.67(1.64$ to 1.70$)$ \\
\hline $70-74$ & $1.94(1.90$ to 1.98$)$ \\
\hline $75-79$ & $2.33(2.28$ to 2.38$)$ \\
\hline Cohort*: & \\
\hline 1896 & $0.84(0.82$ to 0.86$)$ \\
\hline 1901 & $0.86(0.84$ to 0.88$)$ \\
\hline 1906 & $0.91(0.89$ to 0.92$)$ \\
\hline 1911 & $0.94(0.93$ to 0.95$)$ \\
\hline 1916 & $0.95(0.94$ to 0.97$)$ \\
\hline 1921 & 1.00 (baseline) \\
\hline 1926 & $1.02(1.00$ to 1.03$)$ \\
\hline 1931 & $1.00(0.98$ to 1.02$)$ \\
\hline 1936 & $0.96(0.94$ to 0.98$)$ \\
\hline 1941 & $0.93(0.90$ to 0.96$)$ \\
\hline $1946 †$ & 0.90 \\
\hline
\end{tabular}

*Middle year of five year period of birth.

†Model not used for rate predictions because of statistical instability (wide confidence limits). 1946 cohort rate ratio estimated by extrapolating the 1896 to 1941 cohort rate ratios with quadratic model (rate ratios increase to 1926 and decrease thereafter).

Model predicts mortality of 75.1 per 100000 for women aged 50-54 born in 1921 cohort (baseline, sometimes referred to as corner or grand mean). For women aged 75-79 born in 1906 cohort, model therefore predicts rate of $(2.3 \times 0.91 \times 75.1)$ per $100000=159.3$ per 100000 , etc.

8 Duffy SW, Tabar L. Screening for breast cancer [letter]. Lancet 1996; 346:852.

9 Stockton D, Davies T, Day N, McCann J. Retrospective study of reasons for improved survival in patients with breast cancer in East Anglia: earlier diagnosis or better treatment? $B M J$ 1997;314:472-5.

10 Department of Health and Welsh Office. A policy framework for commissioning cancer services. A report by the expert advisory group on cancer to the chief medical offer of Englemd a Whes. London: Department of Heallh, 1995.

11 Clayton D, Schifflers E. Models for temporal variation in cancer rates. I. Age-period and age-cohort models. Stat Med 1987;6:449-67.

12 Chouillet A, Bell CMJ, Hiscox J. Management of breast cancer in southeast England. BMJ 1994;308:168-71.

13 Early Breast Cancer Trialists' Collaborative Group. Systemic treatment of early breast cancer by hormonal cytotoxic, or immune therapy. Lancet 1992;339:71-85.

14 Coleman MP, Esteve J, Damiecki P, Arslan A, Renard H. Trends in cancer incidence and mortality. Lyon: IARC, 1993 (Scientific publications No 121).

15 Langlands AO, Pocock SJ, Kerr GR, Gore SM. Long-tem survival of patients with breast cancer: a study of the curability of the disease. $B M J$ 1979;2:1247-51.

16 Zahl PH, Tretli S. Long-term survival of breast cancer in Norway by age and clinical stage. Stat Med 1997;16:1435-49.

17 Young KC, Wallis MG, Blanks RG, Moss SM. Influence of number of views and mammographic film density on the detection of invasive cancers: results from the NHS breast screening programme. $\mathrm{Br} J$ Radiol 1997;70:482-8

18 Van den Akker-van Marle ME, de Koning H, Boer R, van der Maas P Reduction in breast cancer mortality due to the introduction of mass screening in the Netherlands: comparison with the United Kingdom. Med Screening 1999;6:30-4.

19 Peto R. Five years of tamoxifen-or more? [editorial]. J Natl Cancer Inst 1996;88:1791-3.

20 NHS Breast Screening Programme. NHS BSP 1999 review. Sheffield: NHS BSP, 1999

(Accepted 11 May 2000)

\section{Correction}

Birth characteristics of women who develop gestational diabetes: population based study

A processing error occurred in this article by Grace M Egeland et al (2 September, pp 546-7). None of the reference numbers appear in the text of the printed article, but these can be seen on the $B M J$ website (http://bmj.com/cgi/ content/full/321/7260/546) 